\title{
ARTICLE
}

\section{Clinical neuromodulation in psychiatry: the state of the art or an art in a state?}

\author{
Derek K. Tracy \& Anthony S. David
}

\begin{abstract}
Derek Tracy is a consultant psychiatrist and the associate clinical director for crisis, in-patient, and rehabilitation services at $0 x$ leas NHS Foundation Trust. He runs an MSc module in psychopharmacology at the Institute of Psychiatry, Psychology and Neuroscience (IoPPN), King's College London. Anthony David is Professor of Cognitive Neuropsychiatry at the IoPPN and an honorary consultant psychiatrist at the South London and Maudsley NHS Foundation Trust. Correspondence Dr Derek Tracy, Oxleas NHS Foundation Trust, Green Parks House, Princess Royal University Hospital, London BR6 8NY, UK. Email: derek.tracy@oxleas. nhs.uk
\end{abstract}

\begin{abstract}
SUMMARY
Clinical neuromodulation began in psychiatry with electroconvulsive therapy (ECT), but in recent years several new techniques have been developed: repetitive transcranial magnetic stimulation (rTMS), transcranial direct current stimulation (tDCS), vagal nerve stimulation (VNS), trigeminal nerve stimulation (TNS) and deep brain stimulation (DBS). Each works in a different way, although the principle remains to effect therapeutic change through physically modifying brain activity. Their use in different clinical groups varies between techniques, as does their underlying evidence base. Most support

been the primary example of this in psychiatry, although several other techniques are in current use for a range of conditions. In this article we will describe these less well-established or lesserknown techniques - repetitive transcranial magnetic stimulation (rTMS), transcranial direct current stimulation (tDCS), vagal nerve stimulation (VNS), trigeminal nerve stimulation (TNS) and deep brain stimulation (DBS) - giving an overview of their posited neurophysiological mechanisms of action, their current usage and their existing evidence base. We will conclude by describing the future challenges and necessary work facing this field.
\end{abstract} currently exists for rTMS, with a more modest, but growing database for tDCS. Understandably, but problematically, most research in the other techniques has, to date, been in unmasked open trials. This article describes the mechanism of action and current evidence base for each technique, and notes the challenges facing future work in this potentially important field and new clinical avenue.

\section{LEARNING OBJECTIVES}

- Understand the mechanism of action of rTMS, tDCS, VNS, TNS and DBS

- Be aware of the current clinical uses for each technique and their supporting evidence base

- Appreciate the challenges facing future neuromodulatory research and its potential clinical future

\section{DECLARATION OF INTEREST}

None

Pharmacological and psychological interventions help many people with mental ill health, but they are not effective, or completely effective, for all. They can have side-effects or be undesired by patients, and there is a clear need for novel therapies. Clinical neuromodulation is the physical alteration of cortical or other nervous tissue functioning to effect brain changes and improvements in mental state. Electroconvulsive therapy (ECT) has

\section{Repetitive transcranial magnetic stimulation}

Transcranial magnetic stimulation (TMS) is the most studied of the modern neuromodulatory techniques. It is non-invasive, utilising Faraday's law of induction to alter underlying cortical excitability through an alternating magnetic coil placed over the relevant part of the skull (Barker 1985). Repetitive TMS (rTMS) (Box 1) can be applied in 'slow' low-frequency $(\leq 1 \mathrm{~Hz})$ inhibitory, or 'fast' high-frequency $(\geq 5 \mathrm{~Hz})$ excitatory, paradigms (Pell 2011). Effects on functionally connected regions may be more complex than simple 'inhibition' or 'stimulation' (Tracy 2011) and also potentially dependent on underlying cortical activity levels (Tracy 2014). rTMS effects synaptic

BOX 1 Repetitive transcranial magnetic stimulation (rTMS)

- Non-invasive and painless

- Alternating magnetic coil to the skull can excite or inhibit underlying neurons

- Stronger evidence base in treating depression, slightly weaker for treating hallucinations

- Ongoing experimental work in addictions, eating disorders and self-harm

- A lack of consensus on optimal treatment parameters 
long-term potentiation (LTP) and depression (LTD) (Hoogendam 2010), with animal data showing increased expression of genes important in neuronal plasticity, although overall, rTMSinduced intracellular changes remain incompletely understood.

In psychiatry, rTMS has been most studied in depression and for the treatment of hallucinations in schizophrenia: a relatively recent systematic review of meta-analyses supports its effectiveness in both of these conditions (Hovington 2013). However, it is easy to be overwhelmed by the amount of literature on rTMS and simultaneously be struck by the often conflicting results of trials (Tracy 2014), something that we return to in our conclusions to this article.

\section{rTMS in depression}

In depressive disorders the dorsolateral prefrontal cortex (DLPFC), and connected frontal cortical and deeper limbic regions involved in emotional processing, have been shown to function abnormally; rTMS has been shown to stimulate the hypoactive DLPFC and re-regulate linked regions, including the striatum, thalamus and anterior cingulate cortex (Ridding 2007).

Currently, rTMS depression treatment protocols can vary with respect to several rTMS parameters, although generally they involve fast $(\geq 10 \mathrm{~Hz})$ rTMS to the left DLPFC at $\geq 80 \%$ of the so-called 'motor threshold' (the magnetic strength necessary to elicit activation of the motor cortex - this is affected by several physical variables such as hair and cortical thickness, but is reasonably stable within individuals). Individual sessions typically consist of $4 \mathrm{~s}$ bursts (40 pulses), with $26 \mathrm{~s}$ gaps before the administration of the next pulse sequence; sessions continue for $30 \mathrm{~min}$ or so and deliver about 2400 pulses per session. Treatment protocols usually involve daily rTMS over 2 or 3 weeks.

Most modern rTMS research will have a sham arm with either an rTMS coil that does not deliver a magnetic field, or a protocol that involves tilting an active coil so that the magnetic field does not affect underlying neurons. A meta-analysis of 30 double-blind sham-controlled studies $(n=164)$ using high-frequency rTMS in depression reported an overall mean effect size $d=0.39$ (95\% CI 0.25$0.54)$ for active treatment $(z=6.52, P<0.0001)$ (Schutter 2009). A recent European expert consensus statement on rTMS for psychiatric illnesses graded the existing evidence for highfrequency rTMS to the left DLPFC in depression as 'level A' recommended, namely as having definite antidepressant effect (Lefaucheur 2014).
Repetitive TMS is widely used as a licensed treatment for depression in the USA and across mainland Europe, and is endorsed by the American Psychiatric Association and the World Federation of Societies of Biological Psychiatry. It is not as yet recommended in the UK's National Institute for Health and Care Excellence (NICE) guidelines: a review in 2007 noted that there were no major safety concerns, but stated that there was uncertainty about the clinical effectiveness of rTMS (NICE 2007), and in 2011 NICE determined that there was no need to update this guidance (NICE 2014).

\section{rTMS in schizophrenia}

Treatment protocols for schizophrenia generally involve 'slow' rTMS, specifically targeting and inhibiting auditory verbal hallucinations, on the basis of a neurophysiological model of an overactive speech network during such phenomena (Tracy 2013). The temporoparietal junction is the most common site of application, usually on the left side, although there are also data to support administration to the right. There is a wide range in the duration of individual sessions (from 120 to 2000 pulses) and the number of sessions provided (from 5 to 20). Three meta-analyses have recently evaluated the data on the effectiveness of rTMS in the treatment of auditory verbal hallucinations; the most recent of these (Slotema 2014) demonstrated a mean weighted effect size of rTMS (compared with the sham treatment) of 0.44 , indicating a modest but statistically significant benefit from treatment.

\section{Other uses of rTMS}

Positive therapeutic findings have also been obtained in the treatment of anorexia nervosa (Van den Eynde 2013), bulimia nervosa (Van den Eynde 2010), obsessive-compulsive disorder (OCD) (Mantovani 2010) and depersonalisation disorder (Jay 2014), although individual effect sizes in these less well-studied paradigms are often small and require replication in larger samples. There is also interesting work evaluating the effectiveness of rTMS in altering behaviour such as decision-making - through modification of neuronal circuitry. Inhibitory rTMS to the right DLPFC of healthy volunteers has been shown to induce risk-taking in a gambling paradigm (Knoch 2006), whereas excitatory rTMS to the left DLPFC has been shown to reduce cravings for food (Uher 2005) and cocaine (Camprodon 2007), as well as rates of cigarette smoking (Eichhammer 2003). Speculatively, it has been argued that rTMS could be used to re-regulate frontolimbic disconnectivity that is seen in many affective disorders, including 
emotionally unstable personality disorder, depression and bipolar affective disorder, with the aim of reducing impulsive behaviour such as selfharm (Tracy 2015).

\section{Side-effects of rTMS}

In general, rTMS is well tolerated by participants, with transient headaches and mild discomfort at the site of application being the most common side-effects. There is a potential for inducing a seizure, and, although the absolute risk is low, a history of seizures or traumatic brain injuries are usually exclusion criteria.

\section{Transcranial direct current stimulation}

Transcranial direct current stimulation (tDCS) (Box 2) is a more recent addition to the neuromodulation toolkit. A small current of 1 or $2 \mathrm{~mA}$ produced by a battery is applied to the scalp via two electrodes in saline-soaked sponges. This effects a weak electrical field flowing transcranially between the two electrodes and altering neuronal excitability. Anodal tDCS causes depolarisation and is excitatory; cathodal tDCS induces hyperpolarisation and thus reduces neuronal firing. These changes alter the responses of affected neuronal populations to incoming action potentials beyond the period of tDCS administration, thus putatively modifying long-term neuronal potentiation (Gandiga 2006). Unlike rTMS, current stimulation is continuous, lasting for up to 20 minutes.

Thus far, tDCS has been most studied in depression, with smaller bodies of work exploring effects in schizophrenia and substance misuse, and individual studies looking at OCD and mania. The results in depression are not impressive and certainly not as strong as the rTMS data, although there have been far fewer studies, and only half a dozen or so randomised controlled trials (RCTs). Two meta-analyses evaluating tDCS in treatmentrefractory depression have found conflicting results, although they acknowledge the methodological limitations and small sample sizes of the studies

BOX 2 Transcranial direct current stimulation (tDCS)

- A small current is applied through two electrodes placed on the scalp

- The resultant weak electrical field alters neuronal plasticity

- Relatively cheap equipment, very portable

- Currently a weaker evidence base than rTMS assessed: Kalu et al (2012) determined tDCS to be superior to sham at reducing depression severity in the six RCTs evaluated (Hedges' $g=0.743$, 95\% CI 0.21-1.27); Berlim et al (2013), however, failed to find any benefit for the active treatment in terms of response or remission rates.

In schizophrenia there are emerging data that tDCS might reduce auditory verbal hallucinations (Mondino 2015) and improve cognitive deficits (further details available from the authors on request); early data are also demonstrating enhancement of cognitive performance in healthy participants and post-cerebrovascular accident (Mondino 2014).

Transcranial DCS is generally well tolerated, with itching around the site of electrode placement the most common problem.

In the UK, NICE issued guidance for tDCS for depression in August 2015 (NICE 2015). This noted a lack of major safety concerns, limited data concerning efficacy, and recommended that clinicians wishing to administer tDCS for depression should inform local NHS clinical governance leads.

\section{Vagal nerve stimulation}

Unlike rTMS and tDCS, vagal nerve stimulation (VNS) works on a 'bottom-up' model (Box 3): it stimulates cranial nerves whose nuclei are in the brainstem, which in turn is interconnected with the nuclei of monoaminergic projections and the limbic system. The vagus nerve has somatic, visceral afferent and efferent fibres, and plays an important role in autonomic regulation. Afferent fibres of the vagus nerve innervate the nucleus tractus solitarii bilaterally, and their stimulation with VNS alters the firing rate of noradrenergic neurons in the locus ceruleus and serotonergic neurons in the dorsal raphe nuclei; it has been shown to alter activity in limbic structures, including the amygdala, insula, hypothalamus and thalamus (Nemeroff 2006).

B0X 3 Bottom-up neuromodulation: vagal nerve stimulation (VNS) and trigeminal nerve stimulation (TNS)

- Based on positive mental health findings in epilepsy work

- Act through stimulation of cranial nerves, which in turn alters the ganglia of monoamines in the brain stem

- Produces changes to distal brain regions, including the limbic system and frontal cortex

- Most emerging evidence in depression based on open unmasked trials 
Positron emission tomography (PET) and functional magnetic resonance imaging (fMRI) have further shown that VNS induces more distal effects in regions associated with dysfunction in depression, including the orbitofrontal and anterior cingulate cortices, the right superior and medial frontal cortex; cerebrospinal assays have shown that VNS alters concentrations of monoamines, including serotonin and noradrenaline.

VNS was initially approved for the treatment of refractory epilepsy in the late 1990s. Since work in this population began to show independent improvements in mood (Harden 2000) (fitting with the parallel finding from pharmacological anticonvulsants), it was proposed for use in the treatment of depression. It was approved for adjunctive use in treatment-refractory depression by the Food and Drug Administration in the USA in 2005. In the UK, NICE issued guidance several years ago (NICE 2009) stating that the evidence base for VNS in the treatment of depression was inadequate in quality and quantity, and that it should only be undertaken with special clinical governance arrangements.

\section{Treatment protocol for VNS in depression}

A small watch-size generator consisting of a titanium-encased lithium battery is surgically inserted, under local or general anaesthetic, in the left chest or left axillary region, and a bipolar lead anchored to the left vagus nerve (Landy 1993); the process generally takes less than $1 \mathrm{~h}$. The stimulator is externally operated and intermittently activates the vagal nerve. Exact protocols vary somewhat between studies, but a typical one would involve pulses of about $150-500 \mu$ s activated at a frequency of $20-50 \mathrm{~Hz}$ over $30 \mathrm{~s}$, with a duty cycle of $5 \mathrm{~min}$ 'off' between activations. The current applied varies, gradually increased to test tolerability, with a typical mean of about $0.75-1.00 \mathrm{~mA}$, and maximum of about 3-4 mA. The VNS generator protocol can be altered by computer signalling to the generator, and can be switched on and off telemetrically by moving a hand-held device.

\section{Evidence base for VNS in depression}

Results have varied between trials, although unsurprisingly, given the highly invasive nature of the intervention - it has generally been carried out on individuals with very treatment-resistant depression. For practical reasons, early studies were open, non-controlled trials and they showed promise, with reasonable rates of response and remission in such cohorts. The first, and to date only, shamcontrolled evaluation, a multi-site 3-month RCT comparing VNS with sham stimulation in 235 outpatients with refractory major depressive disorders or bipolar depression showed slightly greater response rates for 10 weeks of the active treatment, but the findings were not significant (Rush 2005). A patient-level $(n>1000)$ meta-analysis (Berry 2013) has shown that in chronic treatment-resistant depression VNS added to treatment as usual had greater response and remission rates, and that these persisted for longer, than treatment as usual alone. The odds of treatment response were three times those for participants who did not receive VNS; the number needed to treat (NNT) was 8 at 12 weeks, dropping to 6 by 96 weeks (compare with an NNT of 5 for ECT in similar cohorts). However, that only six studies were included in this meta-analysis, only one of which was an RCT, gives an indication of the current size of the research area.

\section{Side-effects of VNS}

VNS has generally been a well-tolerated intervention; current findings are consistent with the VNS literature in epilepsy and what might be physiologically anticipated through interference with the superior and recurrent laryngeal nerves. Hoarseness, voice alteration, dyspnoea, pain, an increase in coughing, bradycardia and surgical site pain are the most commonly recorded adverse incidents.

\section{Trigeminal nerve stimulation}

The trigeminal nerve conveys sensory information from the face to the brainstem. Its ganglion is in the cavum trigeminale, and projections to the trigeminal nucleus thereafter reciprocally innervate the nucleus tractus solitarii, locus ceruleus, thalamic structures and reticular formation. Stimulation of the nerve has been demonstrated to alter cortical synchronicity. Like VNS, trigeminal nerve stimulation (TNS) works on a 'bottom-up' model (Box 3) and was originally used in the treatment of epilepsy. Two electrodes just over $25 \mathrm{~mm}$ in diameter are soaked in a saline solution to reduce electrical impedance and placed on the forehead to stimulate the $V_{1}$ branch of the trigeminal nerve bilaterally. Pulses of $250 \mu$ s are applied at a frequency of $120 \mathrm{~Hz}$ in 30 s cycles for varying durations. The current is adjustable, typically up to $100 \mathrm{~mA}$, to ensure that it is well tolerated: this is usually taken to mean mild paraesthesia without muscle contraction.

\section{Evidence base for TNS in depression}

As with VNS, initial work has been open trial, although there are even more limited data for this technique. Proof of concept work by Schrader 
et al (2011) evaluated TNS in five adults with treatment-refractory depression in an 8-week open-label out-patient trial. A patient diary was used to evaluate adherence to the protocol, which estimated over $80 \%$ fidelity to the duration and number of required sessions. Both clinician and patient ratings showed significant improvements over the duration of the study. The intervention was well tolerated, with sub-electrode erythema the main side-effect. More recently, Shiozawa et al (2014) undertook an open trial involving 11 patients with treatment-refractory depression. Unlike Schrader et al, they adapted a protocol based on tDCS work, utilising a single session per day, lasting $30 \mathrm{~min}$, over 2 weeks (ten sessions in total). All showed a clinical response $(\geq 50 \%$ symptom reduction), with all but one going into remission by the trial conclusion. TNS has been certified as complying with European safety directives, but is not licensed for psychiatric use at this time. Any such work would need local clinical governance approval and review.

\section{Deep brain stimulation}

Deep brain stimulation (DBS) involves the reversible, stereotactically guided, deep neurosurgical implantation (usually under general anaesthesia) of two electrodes that will stimulate very specific brain regions. Two stimulators that power these electrodes are implanted subclavicularly and connected to them by subcutaneous extension wires; their batteries need replacement, via further surgery, every few years. Stimulation is usually continuous, although precise parameters can be adjusted. Complications include those possible with any neurosurgical procedure; DBS can also cause neuropsychiatric problems such as anxiety, depression and mania (Cusin 2012).

DBS in neurological disorders, Tourette syndrome and $O C D$

DBS is best established in the treatment of Parkinson's disease, but has also shown effectiveness in dystonia and essential tremor. In neuropsychiatry, DBS has been used to treat severe, medically intractable Tourette syndrome. There have been several case studies and small series, but very few controlled evaluations (contrasting sham stimulation with real stimulation in a crossover design, e.g. Ackermans 2011). Reducing tic severity tends to be the primary focus of treatment, but reduction in frequently co-occurring psychopathology is also seen as a viable target. A systematic review concluded that, despite the lowquality evidence, most studies report beneficial effects, although adverse effects can be significant and careful patient selection is mandatory (Piedad 2012). One factor inhibiting progress, apart from the paucity of controlled trials, is the lack of consensus regarding where the electrodes should be placed (the 'target'), with a case being made for several subcortical sites, most commonly specific regions of the globus pallidus or the thalamus.

DBS has received licensing approval in the USA for the management of refractory OCD, with electrodes applied to the anterior limb of the internal capsule (Nuttin 1999); however, in the UK it remains unlicensed and without regulatory approval for neuropsychiatric indications, although it has been evaluated with some success for Tourette syndrome (Vandewalle 1999).

\section{$D B S$ in depression}

DBS is being trialled in treatment-refractory depression: interpretation of the data is confounded by the fact that the invasiveness and costs of the process mean that it is reserved for very refractory illness, and studies have generally been limited to open trials such as that by Hilimire et al (2015). The Brodmann Area 25 Deep Brain Neurostimulation (BROADEN) study, led by Sidney Kennedy, aimed to compare real and sham DBS in depression, but it was stopped by the Food and Drugs Administration after failing a futility analysis that indicated there was unlikely to be any between-group difference; however, the same group has recently instigated a new placebocontrolled double-blind cross-over of DBS for depression (St Jude Medical 2015).

There is no universal agreement on the single best site to target in depression, but work has variously looked at stimulation of regions known to be involved in the pathophysiology of this illness, with five major targets emerging to date: the subgenual cingulate cortex (Brodmann area (BA) $25)$; the inferior thalamic peduncle; the rostral cingulate cortex (BA 24a); the ventral striatum/ nucleus accumbens; and the lateral habenula.

Overall, the limited open-trial data that do exist have shown varying but, in the clinical circumstances, impressive response levels that are generally over $50 \%$, and remission rates of 30 $50 \%$ 1-2 years post-surgery, with the inevitable caveats such trial methodologies raise. However, the single sham-controlled study undertaken to date did not find any significant improvement in the active stimulation group (Dougherty 2015).

\section{Unanswered questions and future work}

The overall clinical neuromodulation data are mixed, whichever technique is evaluated, although 
the greatest support exists for the best studied of these, rTMS (Box 4). Even a cursory review of the literature will raise obvious questions about how many of these current limitations are fundamental to the technique, and how many are methodological. Certainly, much work is needed to tease out study confounders before more informed opinions about the effectiveness of the techniques can be confidently made. The neurophysiological findings of areas of dysregulation in mental illness are reasonably consistent; the underlying physics and neurophysiology of altering cortical or other neural tissue firing is strong; the therapeutic application and amelioration of symptoms of mental illness through neuromodulation remains weaker.

\section{Research methodology}

Masking

Masking ('blinding') is clearly a potential problem with all neuromodulation research. For rTMS, tDCS (and in the future, with more work, TNS), sham interventions can be given reasonably easily, although there is debate in the field about how truly masked this is (Broadbent 2011), and specific mechanisms for applying sham have varied, with no definitive consensus on the issue. Masking is even more of a problem with the invasive techniques of VNS and DBS, although a way around this, in the very limited number of trials that have tried it, has been to implant an active device in all participants but not to turn it on, and to offer the active treatment afterwards to those initially in a sham arm. Dishearteningly, where this has occurred, initially more promising open trial results have often been tempered by findings in the methodologically more rigorous ones.

Four other major areas need considerable further evaluation: treatment protocols, trial samples, outcome measures and comparison interventions.

\section{Treatment protocols}

For each technique there remains a lack of consensus about the appropriate protocol

\section{BOX 4 Key points}

- Positive data are emerging for all neuromodulatory techniques

- The best evidenced technique is rTMS

- Many studies are hindered by masking issues and a lack of consensus on protocols

- Future work should explore any augmenting effects on psychological interventions paradigm. In rTMS in particular there is no clear agreement - although a loose collection of typical protocols have somewhat organically emerged about where, and indeed how, to site the coil. The frequency or amplitude of administration varies, as do the length of an individual session, the number of sessions and the presence or absence of any follow-up. Trials remain predicated on rather simplistic models of 'stimulation' or 'inhibition', when evaluation of postulated neurophysiology tells one that effects are likely to be more nuanced and involve networks of activity. There is a dearth of neuroimaging work to support or refine clinical outcomes, and the data that are there underline that local effects might produce quite different distal ones (Tracy 2010); at this time, we do not know which brain changes cause, or how they each contribute to, any subsequent clinical effects. Many mental illnesses are long-term relapsing and remitting conditions, and successful pharmacological or psychological interventions can take considerable periods of time, but the clinical neuromodulation techniques are typically given in short bursts over a brief time period of only a week or two. Although this is for pragmatic reasons, it raises the question as to whether this is limiting outcomes and whether less frequent but longer-term interventions might have a role.

\section{Sample selection and size}

A second broad area confounding the literature, and one not unique to neuromodulation, is the variation in participant inclusion criteria and the often small trial sizes, which may hinder their ability to detect change. A follow-on from this is that a one-size-fits-all model has seldom been effective with pharmacological or psychological interventions, and small study sizes hinder our ability to determine when and in whom might neuromodulation work. As is often the case, much work is on individuals with treatment-refractory illness, and while this is clinically understandable, work on those, by definition, non-responders to standard care means they are somewhat atypical, or at least may not represent all patient groups.

\section{Outcome measures}

Third, there is considerable variation in the outcome measures used, which makes crosscomparison and meta-analysis of studies more difficult. Standardisation of these factors, or at least of their reporting, would facilitate collection of larger multi-trial databases that might be mined to elucidate the when and in whom questions, and give a more definitive answer to how fundamentally effective neuromodulation is in various disorders. 
Comparison interventions

Finally, there is the question of choosing a comparison intervention for neuromodulatory treatment. Obviously, there is a sequence from showing benefit over placebo to comparison with the best available treatment for a given condition. However, in depression TMS was compared relatively early in its history with ECT: this narrowed the parameters of its possible treatment effect to a particular circumstance and patient group, which may have set a rather high bar (Eranti 2007).

\section{Practical concerns: acceptability, costs and access}

\section{Acceptability}

How acceptable is neuromodulation to patients? This may of course depend on the severity of their illness, the degree of success or failure of past treatments, and the nature of the proposed intervention. In general, the existing literature shows relatively low drop-out rates, but this is, of course, in people who have already signed up for neuromodulation, and there is no work looking at how acceptable it is to more general populations and at reasons for acquiescing to or refusing neuromodulation. rTMS and tDCS are likely to be the most conceptually acceptable owing to their non-invasive nature and relatively short duration of treatment (although it is far from clear that current paradigm lengths are optimal). VNS and DBS have obvious additional hurdles due to their invasive, surgical nature. A similarly unanswered question is the acceptability of these techniques to clinicians; many may feel somewhat uninformed in the field (an issue this article attempts to redress), and have understandable caution in recommending neuromodulation, even if it were an available option.

\section{Costs and access}

Neuromodulation is not cheap, although costs must be offset against the opportunity costs of untreated mental illness. A new TMS machine will cost tens of thousands of pounds, the exact price escalating with the sophistication of added software and hardware (particularly if precise stereotactic localisation is required), and clinical costs are considerable: a standard depression treatment protocol of 15 daily sessions for one patient would mean that, with inevitable transit and other time slippages, a full-time rTMS clinician (who need not be medically qualified) might 'process' eight new patients every 3 weeks, or approximately 60 patients in a year. A tDCS machine is far cheaper, typically in the range of a couple of thousand pounds, and much more portable than an rTMS unit: once again, clinical administration time might be a limiting factor and, of course, at present its evidence base is weaker than that of rTMS.

US figures estimate costs for just the singleday surgical implantation of VNS at $\$ 40000$ (Cusin 2012), and this work noted that such an intervention would not be covered by most medical insurance schemes. DBS implantation is, unsurprisingly, even more costly because of the complex multidisciplinary work and follow-up required, estimated at $\$ 200000$ per individual for the initial procedure, with battery and other hardware replacements adding considerably to this (McIntosh 2011). However, there is likely to be considerable geographical variation: UK figures from 2013 were notably lower, estimating initial DBS surgery in Parkinson's disease to cost up to $£ 15000$ - to which hardware costs must be added - and a 5-year economic model estimated approximately $£ 20000$ per quality adjusted life year (QALY) (NHS Commissioning Board 2013). Procedural and equipment figures are likely to reduce if the interventions became more widespread, but undoubtedly remain significant at this time.

\section{Unanswered conceptual questions}

Two broad conceptual issues that have not been adequately addressed arise in the clinical neuromodulatory literature. The first is that most of the techniques are principled on altering neural plasticity, but outcomes have generally only been measured through change on clinical scales. Future work might meaningfully evaluate neuromodulation as potentially opening a therapeutic window of enhanced brain plasticity, during which time other interventions could be introduced and augmented by these brain changes. The effects of neuromodulation on enhancing other techniques, such as cognitive remediation, cognitive-behavioural (or other psychological) therapy, or pharmacological intervention, are largely unstudied. In this regard Keefe et al's (2011) evocative metaphor of neuronal plasticity without parallel intervention being akin to consuming protein without exercising is apposite.

A second conceptual issue regards whether or not neuromodulation has been too stuck on diagnostic categories such as depression or schizophrenia, with consequent measurement of improvement using more general symptom scales. Fitting with this, a recent consensus document by a consortium of European experts (Lefaucheur 2014) that evaluated the evidence for rTMS noted 
at various points the underlying brain circuitry putatively modified, but continued to consider future developments in terms of specific illness treatments. It would seem appropriate for the application of neuromodulation to be considered in terms of neuro(patho)physiology and brain circuitry. Such a pan-diagnostic approach offers interesting, novel, testable hypotheses of the effects of neuromodulation on, for example, impulsivity, mood, attentional processing and so forth.

\section{Acknowledegments}

D.T. and A.D. are supported by the National Institute of Health Research Biomedical Research Centre (BRC) at the South London and Maudsley NHS Foundation Trust and the Institute of Psychiatry, Psychology and Neuroscience, King's College London.

\section{References}

Ackermans L, Duits A, Van Der Linden C, et al (2011) Double-blind clinical trial of thalamic stimulation in patients with Tourette syndrome. Brain, 134: 832-44.

Barker AT, Jalinous R, Freeston IL (1985) Non-invasive magnetic stimulation of human motor cortex. Lancet, 1: 1106-7.

Berlim MT, Van Den Eynde F, Daskalakis ZJ (2013) Clinical utility of transcranial direct current stimulation (TDCS) for treating major depression: a systematic review and meta-analysis of randomized, double-blind and sham-controlled trials. Journal of Psychiatric Research, 47: 1-7

Berry SM, Broglio K, Bunker M, et al (2013) A patient-level meta-analysis of studies evaluating vagus nerve stimulation therapy for treatmentresistant depression. Medical Devices, 6: 17-35

Broadbent HJ, Van Den Eynde F, Guillaume S, et al (2011) Blinding success of rTMS applied to the dorsolateral prefrontal cortex in randomised sham-controlled trials: a systematic review. World Journal of Biological Psychiatry, 12: 240-8.

Camprodon JA, Martinez-Raga J, Alonso-Alonso M, et al (2007) One session of high frequency repetitive transcranial magnetic stimulation (rTMS) to the right prefrontal cortex transiently reduces cocaine craving. Drug and Alcohol Dependence, 86: 91-4.

Cusin C, Dougherty DD (2012) Somatic therapies for treatment-resistant depression: ECT, TMS, VNS, DBS. Biology of Mood \& Anxiety Disorders, 2. 14

Dougherty DD, Rezai AR, Carpenter LL, et al (2015) A randomized shamcontrolled trial of deep brain stimulation of the ventral capsule/ventral striatum for chronic treatment-resistant depression. Biological Psychiatry, 78: $240-8$.

Eichhammer P, Johann M, Kharraz A, et al (2003) High-frequency repetitive transcranial magnetic stimulation decreases cigarette smoking. Journal of Clinical Psychiatry, 64: 951-3.

Eranti S, Mogg A, Pluck G, et al (2007) A randomized, controlled trial with 6-month follow-up of repetitive transcranial magnetic stimulation and electroconvulsive therapy for severe depression. American Journal of Psychiatry, 164: 73-81.

Gandiga PC, Hummel FC, Cohen LG (2006) Transcranial DC stimulation (TDCS): a tool for double-blind sham-controlled clinical studies in brain stimulation. Clinical Neurophysiology, 117: 845-50.

Harden CL, Pulver MC, Ravdin LD, et al (2000) A pilot study of mood in epilepsy patients treated with vagus nerve stimulation. Epilepsy \& Behavior, 1: 93-9.
Hilimire MR, Mayberg HS, Holtzheimer PE, et al (2015) Effects of subcallosal cingulate deep brain stimulation on negative self-bias in patients with treatment-resistant depression. Brain Stimulation, 8: 185-91.

Hoogendam JM, Ramakers GM, Di Lazzaro V (2010) Physiology of repetitive transcranial magnetic stimulation of the human brain. Brain Stimulation, 3: 95-118.

Hovington CL, McGirr A, Lepage M, et al (2013) Repetitive transcranial magnetic stimulation (rTMS) for treating major depression and schizophrenia: a systematic review of recent meta-analyses. Annals of Medicine, 45: 308-21

Jay EL, Sierra M, Van Den Eynde F, et al (2014) Testing a neurobiological model of depersonalization disorder using repetitive transcranial magnetic stimulation. Brain Stimulation, 7: 252-9.

Kalu UG, Sexton CE, Loo CK et al (2012) Transcranial direct current stimulation in the treatment of major depression: a meta-analysis. Psychological Medicine, 42: 1791-800.

Keefe RS, Vinogradov S, Medalia A, et al (2011) Report from the working group conference on multisite trial design for cognitive remediation in schizophrenia. Schizophrenia Bulletin, 37: 1057-65.

Knoch D, Gianotti LR, Pascual-Leone A, et al (2006) Disruption of right prefrontal cortex by low-frequency repetitive transcranial magnetic stimulation induces risk-taking behavior. Journal of Neuroscience, 26 : 6469-72.

Landy HJ, Ramsay RE, Slater J, et al (1993) Vagus nerve stimulation for complex partial seizures: surgical technique, safety, and efficacy. Journal of Neurosurgery, 78: 26-31.

Lefaucheur JP, Andre-Obadia N, Antal A, et al (2014) Evidence-based guidelines on the therapeutic use of repetitive transcranial magnetic stimulation (rTMS). Clinical Neurophysiology, 125: 2150-206.

Mantovani A, Simpson HB, Fallon BA, et al (2010) Randomized shamcontrolled trial of repetitive transcranial magnetic stimulation in treatment-resistant obsessive-compulsive disorder. International Journal of Neuropsychopharmacology, 13: 217-27.

McIntosh ES (2011) Perspective on the economic evaluation of deep brain stimulation. Frontiers in Integrative Neuroscience, 5: 19

Mondino M, Bennabi D, Poulet E, et al (2014) Can transcranial direct current stimulation (tDCS) alleviate symptoms and improve cognition in psychiatric disorders? World Journal of Biological Psychiatry, 15: 261-75.

Mondino M, Haesebaert F, Poulet E, et al (2015) Fronto-temporal transcranial direct current stimulation (tDCS) reduces source-monitoring deficits and auditory hallucinations in patients with schizophrenia. Schizophrenia Research, 161: 515-6.

Nemeroff CB, Mayberg HS, Krahl SE, et al (2006) VNS therapy in treatment-resistant depression: clinical evidence and putative neurobiological mechanisms. Neuropsychopharmacology, 31: 1345-55.

National Institute for Health and Care Excellence (2007) Transcranial Magnetic Stimulation for Severe Depression (Interventional Procedure Guidance IPG242). NICE.

National Institute for Health and Care Excellence (2009) Vagus nerve stimulation for treatment-resistant depression (Interventional Procedure Guidance IPG330). NICE.

National Institute for Health and Care Excellence (2014) Guidance. NICE (https://www.nice.org.uk/guidance/ipg242). Accessed 18 September 2015

National Institute for Health and Care Excellence (2015) Transcranial Direct Current Stimulation (tDCS) for Depression (Interventional Procedure Guidance IPG530). NICE.

NHS Commissioning Board (2013) Clinical Commissioning Policy: Deep Brain Stimulation (DBS) in Movement Disorders (NHSCB/D03/P/b). NHS Commissioning Board.

Nuttin B, Cosyns P, Demeulemeester H, et al (1999) Electrical stimulation in anterior limbs of internal capsules in patients with obsessivecompulsive disorder. Lancet, 354: 1526

\section{MCQ answers}

1 a,c 2 b $3 d \quad 4$ a $\quad 5 d$ 
Pell GS, Roth Y, Zangen A (2011) Modulation of cortical excitability induced by repetitive transcranial magnetic stimulation: influence of timing and geometrical parameters and underlying mechanisms. Progress in Neurobiology, 93: 59-98.

Piedad JC, Rickards HE, Cavanna AE (2012) What patients with Gilles de la Tourette syndrome should be treated with deep brain stimulation and what is the best target? Neurosurgery, 71: 173-92.

Ridding MC, Rothwell JC (2007) Is there a future for therapeutic use of transcranial magnetic stimulation? Nature Reviews Neuroscience, 8 : 559-67.

Rush AJ, Marangell LB, Sackeim HA, et al (2005) Vagus nerve stimulation for treatment-resistant depression: a randomized, controlled acute phase trial. Biological Psychiatry, 58: 347-54

Schrader LM, Cook IA, Miller PR, et al (2011) Trigeminal nerve stimulation in major depressive disorder: first proof of concept in an open pilot trial. Epilepsy \& Behavior, 22: 475-8

Schutter DJ (2009) Antidepressant efficacy of high-frequency transcrania magnetic stimulation over the left dorsolateral prefrontal cortex in double-blind sham-controlled designs: a meta-analysis. Psychological Medicine, 39: 65-75.

Shiozawa P, Da Silva ME, Cordeiro 0 (2014) Trigeminal nerve stimulation (TNS) for fibromyalgia: a case study. Epilepsy \& Behavior, 32: 100-1.

Slotema CW, Blom JD, Van Lutterveld R, et al (2014) Review of the efficacy of transcranial magnetic stimulation for auditory verbal hallucinations. Biological Psychiatry, 76: 101-10.

St Jude Medical (2015) A Clinical Evaluation of Subcallosal Cingulate Gyrus Deep Brain Stimulation for Treatment-Resistant Depression (ClinicalTrials.gov NCT01801319). Accessed 30 September 2015.
Tracy DK, O'Daly 0, Joyce DW, et al (2010) An evoked auditory response fMRI study of the effects of rTMS on putative AVH pathways in healthy volunteers. Neuropsychologia, 48: 270-7.

Tracy DK, Ho DK, O'Daly 0, et al (2011) It's not what you say but the way that you say it: an fMRI study of differential lexical and non-lexical prosodic pitch processing. BMC Neuroscience, 12: 128.

Tracy DK, Shergill SS (2013) Mechanisms underlying auditory hallucinations-understanding perception without stimulus. Brain Sciences, 3: 642-69.

Tracy DK, De Sousa De Abreu M, Nalesnik N, et al (2014) Neuroimaging effects of $1 \mathrm{~Hz}$ right temporoparietal rTMS on normal auditory processing: implications for clinical hallucination treatment paradigms. Journal of Clinical Neurophysiology, 31: 541-6.

Tracy DK, Shergill SS, David AS, et al (2015) Self-harm and suicidal acts: a suitable case for treatment of impulsivity-driven behaviour with repetitive transcranial magnetic stimulation (rTMS)? BJPsych Open, 1: 87-91 [open access].

Uher R, Yoganathan D, Mogg A, et al (2005) Effect of left prefrontal repetitive transcranial magnetic stimulation on food craving. Biological Psychiatry, 58: 840-2.

Van den Eynde F, Claudino AM, Mogg A, et al (2010) Repetitive transcranial magnetic stimulation reduces cue-induced food craving in bulimic disorders. Biological Psychiatry, 67: 793-5.

Van den Eynde F, Guillaume S, Broadbent H, et al (2013) Repetitive transcranial magnetic stimulation in anorexia nervosa: a pilot study. European Psychiatry, 28: 98-101.

Vandewalle V, Van der Linden C, Groenewegen HJ, et al (1999) Stereotactic treatment of Gilles de la Tourette syndrome by high frequency stimulation of thalamus. Lancet, 353: 724.

\section{MCQs}

Select the single best option for each question stem

1 Which of the following are 'invasive' neuromodulatory techniques (more than one answer may be correct):

a vagal nerve stimulation (VNS)

b repetitive transcranial magnetic stimulation (rTMS)

c deep brain stimulation (DBS)

$d$ trigeminal nerve stimulation (TNS)

e transcranial direct current stimulation (tDCS).

\section{Practical issues in neuromodulation} include that:

a rTMS should be administered by a medically qualified doctor

b with tDCS, the costs of clinical administration are greater than the cost of equipment

c drop-out rates are generally quite high in neuromodulation research

$d$ trigeminal nerve stimulation requires battery replacement roughly every year

e deep brain stimulation requires the patient to be awake during surgery for appropriate electrode placement.
3 Research in neuromodulation:

a shows that it facilitates psychological therapy through enhanced neuronal plasticity

b cannot in practice be successfully masked because of its invasive/physical nature

c is strengthened by the general use of patients with treatment-refractory illness

$\mathrm{d}$ indicates that appropriate brain stimulation might reduce suicidal acts

e has garnered relatively robust evidence for optimal treatment protocols

4 Repetitive transcranial magnetic stimulation (rTMS):

a can induce seizures

b should be applied via electrodes soaked in a saline solution to reduce impedance

c is delivered through an easily portable device

$\mathrm{d}$ has been shown to reduce risk-taking in healthy volunteers in a gambling task

e is generally applied as a 'fast' excitatory protocol when treating hallucinations.
5 The neuromodulatory treatment of depression:

a has a relatively good evidence base in tDCS

b should be applied in an $8 \mathrm{~h}$ overnight sleeping protocol for VNS

c is typically applied twice a week for six to twelve sessions, analogous to ECT, when rTMS is used

$\mathrm{d}$ is usually through application to the dorsolateral prefrontal cortex in rTMS

e has shown particularly poor results in the early DBS work. 